\title{
« Education, productivité et gain. Retour sur les approches critiques de l'enchaînement causal de la théorie du capital humain »
}

\author{
$\underline{\text { Auteurs }}$ \\ Valérie Canals, Claude Diebolt, Magali Jaoul-Grammare \\ Document de Travail n² $2015-22$
}

Octobre 2015

Faculté des sciences économiques et de gestion

Pôle européen de gestion et d'économie (PEGE) 61 avenue de la Forêt Noire

F-67085 Strasbourg Cedex

Secrétariat du BETA

Géraldine Del Fabbro Tél. : (33) 0368852069 Fax : (33) 0368852070 g.delfabbro @unistra.fr www.beta-umr7522.fr 


\title{
Education, productivité et gain \\ Retour sur les approches critiques de l'enchaînement causal de la théorie du capital humain
}

\author{
Valérie Canals ${ }^{1}$, Claude Diebolt ${ }^{2}$, Magali Jaoul-Grammare ${ }^{3}$
}

\begin{abstract}
Résumé : Cet article analyse les implications économiques de l'éducation pour les individus qui la reçoivent. Nous nous concentrons sur les travaux de référence des théories du capital humain et de ses approches critiques. Notre ambition est de questionner les différentes avancées de la relation éducation-productivité-gain et, en extension, de proposer une première évaluation empirique centrée sur le cas français.
\end{abstract}

Mots clés : capital humain, éducation, filtre, productivité, gain.

Classification JEL : I21, I26, C12

\section{INTRODUCTION}

Quelles peuvent être les implications économiques de l'éducation pour les individus qui la reçoivent ? C'est depuis Adam Smith, en 1776, mais surtout depuis les années 1960 que des efforts importants de réponse à cette interrogation, sous la forme d'une théorisation systématique, avec les contributions décisives de Mincer (1958), Schultz (1961), Becker (1964), etc. vont voir le jour et donner naissance à la théorie du capital humain. Une fois dépassé l'élan suscité par ces travaux fondateurs, les recherches sur ce thème ont marqué le pas durant les années 1970. Mais la persistance de la période de difficultés économiques, ainsi que les nouvelles interrogations sur les ressorts de la croissance ont contribué, vers le milieu des années 1980, à relancer l'analyse économique de l'éducation. Celle-ci est étudiée sous toutes les formes (éducation formelle, formation sur le tas, etc.) et les recherches sont étendues à tous les aspects du développement des ressources humaines (Diebolt, 2014).

A l'origine, la théorie du capital humain appréhendait les dépenses d'éducation comme un investissement dont on cherchait à spécifier la demande et à comprendre l'incidence sur la croissance économique. Les dépenses d'éducation étaient un facteur d'efficacité, qui élevait la productivité et déterminait le niveau et la distribution des gains individuels. Cette analyse a été soumise à différentes critiques (modèle du filtre, théorie radicale, etc.) qui ont engendré de nouvelles pistes de recherche. On s'est ainsi efforcé d'étudier l'offre d'éducation, l'efficacité interne du système d'éducation, le

\footnotetext{
${ }^{1}$ Université Paul Valéry Montpellier. Email: valerie.canals@univ-montp3.fr

${ }^{2}$ BETA/CNRS, Université de Strasbourg, Email: cdiebolt@unistra.fr

${ }^{3}$ BETA/CNRS, Université de Strasbourg. Email: jaoulgrammare@ beta-cnrs.unistra.fr
} 
financement optimal du système de formation, les liens entre éducation et santé, et les analyses économiques de la famille. Depuis les années 1980, l'on s'intéresse aussi à la construction de modèles de croissance qui tentent de mieux représenter les différents aspects des liens éducation/croissance économique.

La diversité même de tous ces travaux rend difficile toute approche exhaustive.

Ce travail se concentre sur les travaux de référence des théories du capital humain et de ses approches critiques. L'objectif est de revenir sur les diverses approches de la relation éducation-productivité-gain.

Notre travail présentera dans un premier temps les théories du capital humain (I) puis les approches critiques (II) qu'elles soient basées sur l'hypothèse du filtre (21) ou sur le rôle du salaire et du marché du travail (22). Enfin, la dernière partie (III) propose une brève évaluation empirique pour le cas français.

\section{LES THEORIES DU CAPITAL HUMAIN}

Le capital humain se définit comme l'ensemble des capacités productives qu'un individu acquiert par l'accumulation de connaissances générales ou spécifiques, de savoir-faire. La notion de capital exprime l'idée que c'est un stock immatériel imputé à une personne pouvant être accumulé et pouvant s'user.

La théorie traditionnelle du capital humain, selon laquelle il existe une relation éducation-productivité-salaire, résulte des contributions initiales de Becker, Schultz et Mincer.

L'hypothèse fondamentale de cette théorie est que l'éducation représente un investissement qui permet l'accroissement de la productivité de ceux qui en bénéficient. Elle implique aussi une augmentation de leurs rémunérations.

La première relation causale stipule que la formation (sous sa forme générale ou spécifique) affecte de manière positive la productivité des individus (Becker, 1964). La production résulte ainsi de la conjonction de trois facteurs :

$$
\mathrm{Y}=\mathrm{f}(\mathrm{K}, \mathrm{L}, \mathrm{H})
$$

où $\mathrm{K}$ est le capital physique, $\mathrm{L}$ le travail et $\mathrm{H}$ le capital humain.

La seconde relation causale résulte du cadre néo-classique selon lequel l'hypothèse de concurrence requiert que les facteurs soient rémunérés à leur productivité marginale. Il s'en suit 3 observations :

- les travailleurs ayant la même productivité marginale, ils sont rémunérés au même taux ;

- les travailleurs les plus productifs sont les mieux payés ;

- les travailleurs les mieux éduqués sont les mieux payés et ils devraient également être les plus productifs.

Toutefois, Maglen (1990) par exemple a soulevé la vulnérabilité de cette seconde relation puisque, très souvent, le marché du travail ne peut être considéré comme concurrentiel. 


\subsection{La théorie de Schulz (1961)}

Pour Schultz « l'investissement en capital humain » est l'explication la plus pertinente de la croissance importante des produits nationaux par rapport à l'augmentation des terres, des heures de travail et du capital physique reproductible.

L'auteur définit par investissement en capital humain les dépenses directes d'éducation, la santé et les migrations internes permettant l'accès à de meilleurs métiers. Néanmoins, aucune d'entre elles ne figure dans les comptes nationaux alors que de nombreux paradoxes concernant la croissance économique peuvent être résolus en prenant en compte cet investissement dans la comptabilité nationale. Se pose alors le problème de savoir ce qu'est un investissement humain et s'il peut être distingué de la consommation.

Schultz distingue trois types de dépenses :

- les dépenses satisfaisant les préférences des consommateurs, mais n'améliorant pas les capacités productives des individus ; il s'agit de la consommation pure ;

- les dépenses améliorant les capacités des individus mais ne satisfaisant pas un besoin de consommation ; c'est l'investissement pur ;

- les dépenses ayant les deux effets.

Malgré les difficultés de mesure exacte de ce que l'on entend par investissement en capital humain, l'auteur distingue parmi les dépenses cinq grands types d'activité ayant un rôle essentiel dans l'amélioration des capacités humaines :

- les moyens et services de santé ;

- l'éducation formelle (primaire, secondaire et supérieur);

- la formation y compris l'apprentissage au sein de l'entreprise ;

- les programmes de formation pour adultes non organisés par l'entreprise ;

- les migrations des individus.

Schultz s'intéresse aux implications que ces activités ont sur les salaires, sur l'amélioration des capacités productives mais également à leurs coûts, notamment ceux de l'éducation.

Pour analyser les effets de la croissance de l'éducation sur les salaires, il est donc nécessaire selon lui, de distinguer le stock d'éducation détenu par la population de la quantité de force de travail. Il choisit de mesurer les connaissances acquises par les individus par l'année scolaire ; il évalue le capital humain par les coûts réels de l'éducation envers l'économie, c'est-à-dire les coûts d'opportunitét et les coûts d'enseignement.

Schultz estime les effets d'un investissement en capital humain. Pour lui, le stock d'éducation contenu dans la force de travail augmente d'environ 8,5 fois entre 1900 et 1956 alors que le stock de capital n'augmente que de 4,5 fois environ. Ainsi durant cette période, l'accroissement de l'éducation représente $17 \%$ de la croissance économique américaine.

\footnotetext{
${ }^{4}$ En choisissant d'étudier, les individus renoncent à des gains immédiats qu'ils pourraient recevoir en échange de leur force de travail.
} 


\subsection{La théorie de Becker (1964)}

L'idée principale de Becker est que certaines activités affectent le bien-être futur par le biais des salaires et de la consommation. Les effets peuvent s'opérer à travers les ressources physiques ou à travers les ressources humaines comme l'éducation.

L'objet de la théorie de Becker est d'étudier les activités influençant le revenu réel grâce à un investissement en ressources humaines et qu'il nomme : «investissement en capital humain ». Ces activités peuvent prendre diverses formes (éducation, formation, soin de santé...), mais elles diffèrent quant à leurs effets sur les salaires et la consommation, à la quantité de ressources investies et par rapport à l'étendue selon laquelle le lien entre investissement et rendement est perçu.

Becker considère trois sortes d'investissement possible :

- La formation générale est courante dans de nombreuses firmes et permet d'accroître la productivité des travailleurs de manière générale si bien qu'elle peut profiter à terme à une firme concurrente. Ainsi, une firme pourrait supporter des coûts de formation sans pour autant en retirer un bénéfice. Dans cette optique, une firme ne fournira une formation que dans le cas où elle n'en supportera pas les frais. Ceux-ci vont alors être à la charge des travailleurs. L'individu qui se forme aura durant cette période un salaire inférieur à sa productivité marginale du fait des coûts de formation.

- La formation spécifique n'est utile qu'à l'entreprise qui la dispense. Il n'est donc pas intéressant pour les travailleurs de la financer puisqu'il leur sera difficile d'en tirer avantage dans une autre entreprise. Les coûts vont donc être supportés par l'entreprise.

- En ce qui concerne l'éducation scolaire, la principale prédiction de la théorie du capital humain est que, dans ce cas, la demande d'éducation au-delà de la scolarité obligatoire est sensible à la fois à des variations de coûts directs et indirects privés de formation et à des variations de gains différentiels liées aux années supplémentaires d'études.

Toutefois, pour que des individus décident de s'éduquer ou de se former, il faut qu'ils perçoivent l'éducation ou la formation comme un investissement rentable, c'est à dire qu'ils vont demander plus d'éducation si le taux de rendement interne anticipé d'une unité supplémentaire de temps de formation est supérieur à celui d'autres investissements à coûts identiques. La demande d'éducation (DE) s'exprime donc comme une fonction croissante de la rentabilité anticipée (r) : DE $=\mathrm{f}(\mathrm{r})$ avec $\partial f / \partial r>0$.

Suite à cette formulation, diverses critiques ont été énoncées. Tout d'abord l'éducation ne constitue pas uniquement un investissement. Elle peut également être considérée comme un bien de consommation (Schultz, 1963). Par ailleurs, le modèle ne prend pas en compte d'autres facteurs pouvant influencer la demande d'éducation comme le taux de chômage, la région, le sexe (Psacharopoulos et Woodhall, 1988), les facteurs sociaux (Anderson, 1983) ou le risque d'échec (Eicher \& Mingat, 1982). 
L'un des moyens naturels pour tester la théorie du capital humain est le calcul des taux de rendement de l'éducation.

\subsection{La formalisation de Mincer}

Puisque la théorie orthodoxe du capital humain débouche sur la conclusion que les individus sont rémunérés à leur productivité marginale, reflet de leur capital humain, la quantité et la qualité de formation reçue apparaît comme un déterminant essentiel des gains de l'individu. Ainsi, comme le suggère Becker (1975), les différences de gains entre individus proviennent de leur éducation.

Sous les hypothèses suivantes, Mincer va spécifier de manière économétrique la relation précédente :

- tous les individus ont des capacités et des opportunités identiques d'occuper un poste de travail ;

- les postes de travail diffèrent dans la quantité de formation qu'ils requièrent ;

- la formation prend du temps et chaque année de formation entraîne un report de salaire à une année ultérieure ;

- la quantité de revenu est stable pendant la durée de vie active.

Il en résulte que les salaires annuels correspondant aux différents niveaux de formation, diffèrent de manière multiplicative.

Si l'on minore la première hypothèse, il existe une corrélation positive entre la quantité de formation et les capacités; dans ce cas, les personnes les plus aptes à apprendre choisiront une formation plus longue. Ainsi, le revenu dépend des qualités individuelles. Mincer propose deux formes complémentaires d'acquisition du capital humain :

- l'investissement scolaire : hypothèse selon laquelle le capital humain est acquis exclusivement lors de la scolarité ;

- l'investissement en expérience professionnelle. Extension de l'hypothèse précédente, l'individu continue d'investir en capital humain au cours de sa vie active. Comme cette acquisition est coûteuse, le salaire observé diffère du salaire anticipé.

Donc, au lieu d'observer un niveau de salaire constant avec l'âge, on observe un profil âge/salaire croissant. Le salaire continuera à augmenter tant que l'investissement net va croître à un taux inférieur au taux de rendement des investissements professionnels.

Sous l'hypothèse de décroissance linéaire de l'intensité des investissements professionnels et de la constance du rendement de ces investissements, Mincer montre la relation suivante :

$$
\operatorname{Ln} \mathrm{Y}=\mathrm{a}+\mathrm{b} . \mathrm{S}+\mathrm{c} \cdot \mathrm{E}-\mathrm{d} \cdot \mathrm{E}^{2}+\mathrm{v}
$$


Y représente les gains.

S est l'éducation formelle, la scolarité.

E l'expérience professionnelle, mesurée par l'âge de l'individu.

Ainsi, plus le capital humain général (S) et spécifique (E) sont élevés, plus les gains sont importants ceteris paribus. En conséquence, les individus vont investir de moins en moins dans la formation à mesure que se déroule leur vie professionnelle.

On peut également prendre en considération le fait que le rendement marginal varie avec le volume des investissements éducatifs :

$$
\operatorname{Ln} Y=a+b \cdot n+c \cdot n^{2}+\text { d.E }- \text { e. } E^{2}+v
$$

Où $\mathrm{n}$ est le nombre d'années d'études avec un taux de rendement marginal $(\partial \ln Y / \partial n)=b+2 c n$ dans lequel on anticipe $c<0$, matérialisant des rendements marginaux décroissants.

Le modèle suggère que la distribution de gain d'une société reflète les niveaux d'éducation. De manière générale, on peut conclure que pour réduire les disparités des niveaux d'éducation, il faut réduire les disparités nationales ou internationales de revenu. Toutefois, dans de nombreux pays, à niveau de formation donné, des différences de gain persistent selon la race et le sexe.

Si pour la théorie du capital humain, les différences de gain entre communautés raciales sont expliquées par les inégalités de quantité et de qualité d'éducation qui les caractérisent, l'étude du différentiel de gain selon le genre est plus variée. Pour Polachek $(1974,1978)$ il est le résultat d'une attitude particulière des femmes en termes d'offre de travail -l'hypothèse d'atrophie- selon laquelle les individus participant à l'activité économique de manière discontinue optent pour une moindre accumulation du capital humain. Goldin (1986) rejoint cette hypothèse en stipulant que la faiblesse des gains des femmes s'explique par une rotation de la force de travail plus importante que celle des hommes. En revanche, Becker (1985) pense que les femmes gagnent moins que les hommes en raison d'un choix de métier leur permettant de conserver une part de leur énergie pour les travaux domestiques. Il rejoint ici l'idée de Filer (1986) qui pense que la différence provient d'une différence de fonction d'utilité.

La théorie du capital humain et les modèles qui en découlent ont fait l'objet de nombreuses vérifications empiriques, tant sur le plan de l'impact global de l'éducation sur la croissance économique, qu'au plan individuel du lien entre éducation et productivité par exemple.

Suite aux travaux de Schultz $(1961,1962)$ et Denison (1962), qui montrent que la croissance économique des Etats Unis est due pour une part non négligeable à l'accroissement de l'éducation de la force de travail, de nombreux auteurs (Kruger, 1968), (Selowsky, 1969), (Griliches, 1970), (Psacharopoulos, 1973 et 1984), (Daly, 1982), (Jorgensen, 1984), (Plant \& Welch, 1984), (Jorgensen et Fraumeni, 1992) etc. vont appliquer leur méthode à divers pays. Si la plupart des résultats rejoignent les 
premiers, ils différent dans le temps et dans l'espace et selon la nature des pays (industrialisés ou en voie de développement). De plus, à partir de données sur l'Australie, Maglen (1990) montre que pour les périodes 1968-1973 et 1973-1979, malgré un accroissement de l'investissement global en éducation, la productivité et le PIB ont connu un déclin.

Les études empiriques de ce lien ne donnent pas de résultats unanimes. Si Lockheed et alii (1980) puis Jamison et Lau (1982) montrent que l'éducation a un effet positif sur la productivité des agriculteurs, Mook (1981) et Gurgand (1993) trouvent des résultats contraires. En considérant des secteurs d'activités différents, Berg (1970), Layard et alii (1971), et Bery (1980) n'ont pu trancher sur la nature de la relation entre éducation et productivité.

En ce qui concerne la liaison entre productivité et gain, les études réalisées sont moins nombreuses que les précédentes; elles sont en revanche uniformes quant à leurs résultats. Ainsi, Gattchalk (1978), Abrahams \& Medoff (1980, 1981), Maranto \& Rodgers (1984), Rumberger (1987) et Weiss (1988) concluent tous à un effet non significatif de la productivité sur les revenus des travailleurs.

La relation de la théorie du capital humain la plus testée est la corrélation gain/éducation ; elle demeure à ce jour confirmée (Wagner, 1990 ; Sofer, 1990).

\section{LES THEORIES CRITIQUES DE LA THEORIE DU CAPITAL HUMAIN}

L'enchaînement causal éducation - productivité - gain prôné par la théorie du capital humain a suscité de nombreuses controverses et donné naissance à des théories rivales. Elles prennent principalement en compte la spécificité du marché du travail et se déclinent selon plusieurs axes selon que leurs auteurs partagent tout ou partie des hypothèses néo-classiques de la théorie du capital humain.

D'une part, les théories basées sur l'hypothèse du filtre (Berg, 1970 ; Phelps, 1972 ; Thurow, 1972 ; Spence, 1973 et Arrow, 1973) adaptent l'hypothèse du filtre à l'analyse du marché du travail en supposant que les employeurs sont en situation d'incertitude quant aux capacités productives des candidats à l'embauche.

D'autre part, les théories non orthodoxes (Doeringer et Piore, 1971; Cain, 1976 ; Akerlof, 1984) se distinguent des théories précédentes par le fait qu'elles basent leur analyse sur les groupes et non sur les individus.

\subsection{Les théories basées sur l'hypothèse du filtre}

\subsubsection{Le modèle de la discrimination statistique de Phelps (1972)}

Initialement destiné à expliquer les différences de gains entre race et sexe, ce modèle s'est ensuite étendu à l'analyse économique de l'éducation.

Les employeurs pensent que les travailleurs les plus éduqués sont les plus productifs. Ils vont donc réserver les emplois les plus rémunérés aux travailleurs les 
plus formés. Toutefois, ils opèrent dans un univers incertain où les informations sur la productivité potentielle des travailleurs sont coûteuses.

Face à l'imperfection de l'information sur les caractéristiques des candidats à l'embauche, les employeurs vont s'appuyer sur des indicateurs statistiques reflétant les performances moyennes des catégories de référence pour sélectionner leurs futurs employés.

La rémunération des employés ne serait pas le résultat d'une productivité effective, mais plus celui d'une discrimination de la part des employeurs basée sur le niveau d'éducation.

\subsubsection{Le modèle de signalement de Spence}

Supposant l'existence d'une corrélation positive entre productivité et qualification, ce modèle stipule que les employés les plus éduqués devraient percevoir un salaire plus important. C'est une interprétation alternative de la corrélation entre salaire et formation, mais où le salaire reflète les aptitudes des individus signalées à l'employeur par la réussite académique.

Dans la plupart des marchés du travail, l'employeur ignore les capacités productives d'un individu au moment où il l'embauche. Embaucher quelqu'un équivaut alors à acheter un billet de loterie dont le prix est le salaire qui sera versé ultérieurement à l'employé.

Si la productivité marginale du candidat ne peut être observée avant l'embauche par l'employeur, il peut toutefois observer un éventail de données caractéristiques de l'individu (éducation, métier précédent, casier judiciaire...) qui doivent lui permettre d'évaluer le prix de la loterie.

Après l'embauche, l'employeur découvre les réelles capacités productives de l'individu. Compte tenu de son expérience, l'employeur pourra estimer la probabilité de la productivité conditionnellement aux signaux perçus. Ces derniers apparaissent ainsi comme des paramètres de la probabilité.

Si certains signaux encore appelés indices (sexe, race...) ne peuvent faire l'objet d'aucune manipulation de la part des individus, les véritables signaux peuvent être manipulés par les individus moyennant une certaine somme. On parle alors de coût de signalement. Pour le cas de l'éducation, un individu investira s'il y a un rendement suffisant.

Afin de maximiser la différence entre les salaires offerts et les coûts de signalement, les individus vont opérer une sélection entre les différents signaux. Toutefois, à moins que les coûts de signalement ne soient négativement corrélés à la capacité productive, le signal ne permet pas de distinguer un candidat d'un autre. 
(1972)

2.1.3. Le modèle de la queue pour l'emploi ou de la file d'attente : Thurow

Ce modèle s'appuie sur la notion de concurrence entre travailleurs et repose sur l'idée que les fondements de la productivité résident, d'une part dans le degré d'adaptation des travailleurs à l'entreprise au poste de travail occupé et d'autre part, à la fois dans la formation spécifique offerte par les employeurs et dans les capacités des travailleurs à assimiler ces formations.

Pour les employeurs, le niveau d'éducation des candidats à l'embauche reflète les capacités des individus utilisées lors de la sélection. Les travailleurs vont former une file d'attente dans laquelle figurent au premier rang les candidats les plus éduqués. Ainsi les travailleurs les plus éduqués initialement continueront, ceteris paribus, à accumuler du capital humain afin de rester à la tête de la queue.

Le marché du travail chez Thurow n'est pas un marché des qualifications, mais un marché de la formation où les postes de formation sont attribués aux différents travailleurs. Le coût de la formation étant inconnu de l'employeur, il utilisera des signaux (le niveau d'éducation) comme reflétant l'aptitude à être formé.

\subsubsection{La théorie du filtre (Arrow, 1973) et du parchemin (Berg, 1970)}

La théorie du filtre est la formalisation d'une hypothèse proposée au préalable par le sociologue Berg (1970) et appelée « Théorie du parchemin ».

Cette théorie suppose deux individus, A et B ayant terminé le même parcours scolaire, mais seul l'individu A détient le diplôme X correspondant. Ainsi, bien que leurs acquis soient proches, ceux de l'individu B ne sont pas reconnus et ce dernier est classé avec les individus du diplôme immédiatement inférieur (X -1).

Le diplôme $\mathrm{X}$ produit donc un effet de réduction de l'offre sur le marché des diplômés $\mathrm{X}$ contre un accroissement de l'offre sur celui des diplômés (X -1). On s'éloigne alors de l'hypothèse concurrentielle, dans la mesure où une fraction de l'écart de rémunération entre les deux groupes est due, non pas à la différence de productivité, mais à un effet de répartition du revenu créé artificiellement par le diplôme.

Deux éventualités sont alors envisageables :

- si, après l'embauche, l'employeur peut mesurer les productivités effectives, alors les fonctions de gains des individus de productivité réelle comparable se rapprochent avec l'ancienneté et ce, quel que soit leur diplôme ;

- si l'employeur ne peut que comparer les productivités moyennes, il ne modifiera pas ses anticipations.

On peut donc conclure que l'écart de rémunération entre les groupes sera d'autant plus grand que l'effet de seuil du diplôme est grand. Ceci se traduit au niveau politique par la multiplication des diplômes intermédiaires afin de réduire l'effet de parchemin. 
La théorie du filtre de Arrow (1973) se démarque de la vision orthodoxe par le fait que la condition de transparence sur le marché du travail n'est pas supposée remplie. En effet, l'employeur n'achète pas une productivité connue, mais sélectionne à l'embauche des travailleurs dont la productivité potentielle est inconnue.

Pour que le marché du travail se rapproche des conditions de concurrence, il faut que les employeurs aient une information sur les facultés productives des candidats à l'embauche. Cette information est fournie par le diplôme. Autrement dit, la fonction des établissements scolaires n'est pas le développement des capacités productives, mais la révélation de ces capacités grâce au filtre des diplômes.

Si de nombreuses hypothèses impliquent que l'éducation offre des connaissances qui amènent une productivité supérieure (Théorie du capital humain), Arrow a une vision différente: une éducation supérieure ne contribue en aucun cas à des performances économiques supérieures ; elle n'accroît pas non plus la socialisation. Au contraire, l'enseignement supérieur sert de système de sélection qui sépare les étudiants selon leurs aptitudes.

Cette théorie ne se démarque pas totalement de la théorie du capital humain tout en étant distincte de cette dernière. Cette théorie est basée sur l'hypothèse que les agents économiques ont une information très imparfaite: en particulier l'employeur a une faible idée de la productivité des personnes qu'il recrute.

Arrow suppose au contraire :

- que l'employeur a une très bonne information statistique mais rien d'autre ;

- que certaines informations sur le travailleur, comme les diplômes qu'il possède, peuvent être acquises par l'employeur à un certain coût.

L'employeur connaît, à partir d'informations générales ou d'expériences passées, la distribution statistique des productivités, mais il ne peut connaître les productivités des individus à partir de cette même information. L'employeur ne connaît la productivité de ses employés qu'à partir d'un certain temps.

Selon Arrow, un individu a trois caractéristiques :

- sa scolarité avant l'enseignement supérieur ;

- sa probabilité de réussite dans l'enseignement supérieur ;

- sa productivité.

Ces dernières sont corrélées positivement.

Les employeurs savent seulement si la personne possède un diplôme ou non.

Les établissements d'enseignement supérieur servent en réalité de double filtre

- en sélectionnant les entrants ;

- en faisant réussir ou échouer les étudiants.

Ainsi, un diplôme de l'enseignement supérieur apporte une information: la productivité d'un quelconque diplômé de l'enseignement supérieur est supérieure à celle d'un membre quelconque de la population.

Si l'enseignement supérieur a une valeur informationnelle positive, cela ne signifie pas forcément que cela soit socialement favorable. 
Le modèle du filtre amène ici une conclusion très différente de la théorie du capital humain en mettant en évidence la divergence entre une demande privée d'information et une demande sociale.

Si l'on considère un modèle de production où les individus sont parfaitement substituables dans des proportions données par leur productivité, alors une information sur la productivité n'aura aucune valeur sociale. Les individus les plus productifs ne produisent plus que si quelqu'un peut parfaitement les identifier. Cependant, il y aura une valeur privée du diplôme pour ceux qui ont le plus de chance de posséder celui-ci. Ainsi, le salaire d'un individu sera la valeur espérée de sa production conditionnellement à l'information disponible pour l'employeur.

\subsection{Les théories basées sur le rôle du salaire et du marché du travail}

Ce second courant d'analyse critique tente de montrer que les liens entre formation et salaire sont également très dépendants du marché du travail, par la situation dans laquelle se retrouvent les individus mais également par la nature même du marché du travail.

2.2.1. La théorie des salaires efficients (Akerlof, 1984).

Dès 1957, Leibenstein postule une liaison positive entre le salaire et la productivité des individus. Appliquée au départ aux pays en développement (Mirrlees, 1975 ; Stiglitz, 1976), cette théorie a ensuite été développée par Akelof (1982 ; 1984). Il avance l'idée selon laquelle les entreprises peuvent avoir un intérêt à verser aux employés des salaires supérieurs aux salaires de l'équilibre concurrentiel.

L'argument avancé par Akerlof est que l'efficacité productive d'un salarié dépend essentiellement du sentiment qu'il a d'être «bien traité par son employeur. Ainsi, offrir un salaire supérieur au salaire de réservation constitue une sorte de gratification susceptible d'inciter les individus à augmenter leur niveau d'effort.

Ces salaires, appelés salaires d'efficience, se justifient de diverses manières :

- ils incitent les employés à l'effort, hypothèse de shirking de Bowles (1985) et permettent une réduction des coûts d'encadrement.

- ils permettent aux entreprises d'attirer les travailleurs les plus productifs (Stiglitz, 1976).

- ils permettent à l'entreprise de diminuer le taux de rotation de la main d'œuvre (Stiglitz, 1982).

Bien que l'hypothèse de corrélation positive entre productivité et salaire soit toujours présente, cette théorie se détache de la théorie de base du capital humain en la dépassant. Elle précise tout d'abord que la formation générale n'est plus le seul déterminant de la rémunération. Ensuite, les salaires versés aux employés identiques ne sont plus uniques; ils varient selon l'entreprise, par exemple en fonction des coûts de rotation de la main d'œuvre. Enfin, et c'est essentiellement sur ce point que cette théorie se démarque de la théorie du capital humain, on ne se situe plus dans la logique concurrentielle du capital humain. 
Par ailleurs, la relation causale productivité-gain apparaît inversée : les gains les plus élevés induisent des productivités plus élevées par réduction de la paresse et du « turnover».

Du point de vue empirique, diverses vérifications ont été apportées. Lazear \& Moore (1984) par une analyse comparée des profils âge-gain de deux groupes de travailleurs, montrent qu'il existe une liaison plus significative chez les salariés que chez les travailleurs à leur compte, ce qui semble confirmer que les incitations des employeurs ont un effet notable sur les gains au fur et mesure que l'employé présente une grande ancienneté. Par la suite, Plassard \& Tahar (1990), ont confirmé le modèle des salaires efficients; ils montrent en effet que plus la concentration technique des secteurs est élevée, plus l'entreprise est de grande taille et plus les salaires doivent être élevés pour favoriser l'efficacité.

\subsubsection{Les théories de l'hétérogénéité du marché du travail}

Elles reposent sur l'existence de plusieurs marchés du travail et se divisent en deux classes selon le degré de perméabilité accordé aux marchés du travail.

D'une part, la théorie de la segmentation du marché du travail (Cain, 1976) suppose la présence de plusieurs marchés du travail cloisonnés et imperméables entre eux : le marché primaire et le marché secondaire.

Le premier représente un marché des emplois stables à haute rémunération. Il se divise en deux sous marchés : (I) le segment primaire indépendant ou supérieur qui regroupe les emplois situés en «haut de l'échelle » nécessitant autonomie, capacité à l'innovation et pouvoir de décision et (II) le segment primaire secondaire qui concerne les emplois requérant des qualifications moyennes associées à des rémunérations relativement élevées et des possibilités de promotion.

Le marché secondaire regroupe les emplois nécessitant peu de qualification, avec de faibles possibilités de promotion et associés à des rémunérations faibles. Une des caractéristiques de ce marché est qu'il présente une forte mobilité externe.

A la différence de la théorie du capital humain, cette théorie tend à montrer que l'éducation n'est pas l'unique déterminant à l'embauche et qu'elle agit différemment selon le marché considéré. En effet, sur le marché primaire, l'expérience, l'ancienneté et les règles administratives jouent un rôle plus important dans l'embauche que l'éducation formelle. Ainsi, si la relation qualification - productivité - gain demeure, comme dans la théorie du capital humain, elle est élargie dans le sens où c'est plus l'éducation spécifique qui joue un rôle.

D'autre part, la théorie des marchés de travail internes de Doeringer et Piore (1971), distingue le marché interne du marché externe du travail, les deux n'étant pas totalement imperméables mais reliés par certains emplois qui constituent l'accès au marché interne. 
L'idée centrale de cette théorie et que sur le premier, les salaires se forment sur la base de considérations administratives alors que dans le second, ils sont déterminés par le jeu de l'offre et de la demande. Il existe alors deux types d'emplois : les emplois qui peuvent être pourvus à partir du marché externe et les emplois réservés au marché interne pour lesquels l'accès est régi par la promotion ou les mutations d'employés déjà postés.

Cette théorie s'éloigne de la théorie du capital humain sur plusieurs points :

- l'éducation n'apparaît plus comme l'unique critère d'accès aux postes de travail ;

- l'organisation au sein de l'entreprise affecte la concurrence sur les deux types de marché et influence par la même les salaires qui ne sont plus le parfait reflet des productivités intrinsèques des individus ;

- l'accent est essentiellement mis sur la demande de travail contrairement à la formulation de base qui met l'accent sur l'offre.

On retrouve ici une autre formulation du modèle de la file d'attente de Thurow où le diplôme peut s'analyser comme une forme de rationnement de l'accès à l'emploi dans le sens où le recours à d'autres modes d'accès à l'emploi crée une compétition limitée entre individus.

Les études empiriques de cette théorie réalisées à ce jour (Rao \& Datta, 1985), (Maxwell, 1987), (Hartog, 1987) n’ont pas infirmé la théorie. Les premiers ont montré que l'organisation de l'entreprise exerce une influence significative sur les gains et que l'éducation n'est pas le seul déterminant du salaire. Ces résultats sont rejoints par Hartog qui montre, d'une part que les variables d'offre et de demande du marché du travail exercent simultanément une influence notoire sur les gains mais aussi que pour un niveau d'éducation et d'expérience donné, on n'observe pas une égalisation des salaires pour les différents emplois.

Enfin, Perrot et Zylberberg(1989) formalisent la théorie du salaire efficient sur un marché segmenté à la Doeringer et Piore et montrent que le niveau de salaire primaire doit satisfaire une condition incitative. En effet, ce sont les conditions d'efficacité qui caractérisent l'équilibre entre emploi primaire et l'ampleur du chômage alors que dans le secteur secondaire, l'équilibre est fonction des conditions de migrations entre les deux segments. La segmentation du travail apparait ainsi comme un facteur déterminant dans la détermination des salaires et son association à la théorie du salaire d'efficience tend à «concurrencer, voire éclipser la théorie des contrats implicites (Stiglitz (1984) dans l'explication de l'imparfaite flexibilité des salaires et d'un chômage persistant» (Perrot et Zylberberg, 1989, p. 5).

2.2.3. La théorie du « job competition » de Thurow (1975)

Cette théorie développée par Thurow en 1975 s'oppose radicalement à l'hypothèse du filtre et à la théorie du capital humain. 
Dans la situation où l'emploi est rationné, les individus ne vont plus arbitrer entre le taux de rendement de l'éducation et celui des investissements en éducation pour décrocher un emploi, c'est à dire arrêter leurs études pour prendre un emploi rémunéré à la productivité atteinte, mais vont devoir présenter un niveau de diplôme supérieur à celui des concurrents.

Dans ce cas, seul le meilleur candidat obtiendra un emploi, les autres perdant le bénéfice de leur diplôme. On retrouve ici «le dilemme du prisonnier». Pour un individu, la perte attachée à l'arrêt des études, si les autres continuent, est telle que tous les individus vont choisir de poursuivre leurs études. Le manque à gagner est d'autant plus faible par rapport au gain potentiel que les seules alternatives pour les individus sont le chômage ou un emploi mal rémunéré. Il en résulte une inflation de la demande d'éducation et puisque l'emploi ne croît pas au même rythme et une dévalorisation continue des diplômes.

Les implications politiques sont ainsi différentes de celles des théories précédentes :

- $\quad$ il n'existe pas de mécanisme spontané d'équilibre diplôme - salaire mais un déséquilibre cumulatif ;

- l'effet «boule de neige » de la demande d'éducation est alimenté par le libre accès aux études ;

- l'impossibilité d'entente entre individus engendre l'intervention de l'état comme la seule solution pour freiner la course aux diplômes et pour reconnaître leur hiérarchie ;

En prolongement, d'autres théories plus radicales encore, se sont développées face à la théorie du capital humain.

\subsubsection{Les théories radicales}

S'inspirant des théories marxistes, Bowles \& Gintis $(1975,1976)$ prônent l'idée que l'éducation accroît la productivité en reproduisant la structure de classe de la société et non pas en augmentant leurs qualifications.

Ils supposent ainsi que l'enseignement dispensé aux enfants d'ouvriers, et qui se compose généralement du seul enseignement obligatoire, va se limiter à l'instruction des attitudes nécessaires aux tâches ouvrières. En revanche, l'enseignement supérieur, généralement réservé aux enfants des classes moyennes et supérieures, dispense une éducation nécessaire aux tâches de direction et d'innovation.

Ceci a diverses implications : tout d'abord, les enfants de la classe ouvrière vont être contraints d'occuper les emplois les moins rémunérés et les moins désirés car ils auront accumulé moins de capital humain. Ensuite, dans ce type de système, l'enseignement est au service de l'instance dominante.

Bien que difficiles à tester, ces théories ont pu être vérifiées empiriquement: Kiker \& Heath (1985) ont montré que l'origine sociale des travailleurs a un impact significatif sur leurs gains; Anderson (1983) conclut que les facteurs sociaux exercent un effet sélectif sur l'accès à l'enseignement notamment secondaire et supérieur. 
La discrimination selon l'origine sociale n'est pas la seule présente sur le marché du travail ; il existe aussi une discrimination selon la race ou le sexe.

\section{QUEL ROLE POUR L'EDUCATION EN FRANCE?}

L'objectif de cette dernière partie est de confronter empiriquement la théorie du capital humain aux théories qui lui sont complémentaires dans l'estimation du salaire individuel.

Le but n'est pas de rejeter tel ou tel modèle, mais de caractériser la nature de l'impact de l'éducation et plus particulièrement de l'enseignement supérieur sur le salaire individuel, en déterminant quel modèle -capital humain ou filtre- explique le mieux les salaires des individus.

Pour cela, nous évaluons empiriquement la théorie du capital humain ainsi que trois des théories basées sur l'hypothèse du filtre : la théorie du signal de Spence, la théorie du parchemin de Berg, et la théorie du filtre de Arrow. Nous utilisons la base de données du Cereq (Génération 98) sur l'insertion professionnelle des jeunes sortants de l'enseignement en 1998. Un tri de l'échantillon a conduit à ne garder que les sortants de Génération 98 ayant un niveau de formation supérieur au niveau IV qui sont en emploi et à temps plein à la date de l'enquête. L'échantillon regroupe 12730 individus.

\subsection{Evaluation empirique des théories de Mincer et Arrow}

La théorie du capital humain est évaluée à partir de la formalisation du modèle de scolarité de Mincer en régressant le logarithme du salaire Y sur le nombre d'années de scolarité S de l'individu :

$$
\operatorname{Ln} Y i=a+b \cdot S i+\varepsilon i
$$

Afin d'évaluer le modèle de Arrow, on régresse le logarithme du salaire $\mathrm{Y}$ en fonction de la position $\mathrm{P}$ de l'individu dans sa génération :

$$
\operatorname{LnYi}=a+b . P i+\varepsilon i
$$

En effet, si l'on fait l'hypothèse que la seule valeur de l'éducation reçue, sur le marché du travail, est le signal émis filtré par le système scolaire, on peut affirmer que ce n'est plus la scolarité d'un individu qui détermine ses gains mais la position relative que lui confère sa scolarité dans la distribution du niveau éducatif des individus de sa génération.

Pour estimer la position de l'individu au sein de sa génération, on s'inspire des travaux de Jarousse et Mingat (1986) et de Kroch \& Sjoblom (1994). La position de l'individu $\mathrm{i}$ dans sa génération $\mathrm{P}_{\mathrm{i}}$, est estimée à partir de la distribution du nombre d'années d'études dans chaque classe d'âge $\mathrm{t}[\mathrm{n}(\mathrm{t}) ; \sigma(\mathrm{t}))$, et correspond à la valeur centrée réduite de la scolarisation de l'individu i : $P_{i}=\left(n_{i}-\bar{n}\right) / \sigma_{n}$, avec $\mathrm{n}_{\mathrm{i}}=$ nombre 
d'années d'études de l'individu i, $\bar{n}=$ nombre moyen d'années d'études et $\sigma_{\mathrm{n}}=$ écart type.

L'estimation des deux modèles (Tableau 1) montre que le modèle de Mincer a un pouvoir explicatif plus important que le modèle de Arrow. Ainsi, pour les jeunes diplômés français, la durée de scolarité semble être plus déterminante dans le salaire que la position de l'individu dans sa génération.

Tableau 1. Estimation des modèles de Mincer et Arrow

\begin{tabular}{|c|c|c|c|c|}
\hline & \multicolumn{2}{|c}{ Arrow } & \multicolumn{2}{c|}{ Mincer } \\
\hline Ensemble & 0,1092 & 0,1211 & 0,1025 & 0,2642 \\
\hline Garçon & 0,1416 & 0,1824 & 0.1122 & 0.3188 \\
\hline Fille & 0,0862 & 0,0903 & 0.0916 & 0.2257 \\
\hline
\end{tabular}

\subsection{Evaluation empirique de la théorie du signal de Spence.}

Selon Spence, le salaire dépend du signal émis par la possession d'un diplôme. Afin d'évaluer ce modèle, nous estimons le logarithme du salaire en fonction des divers niveaux de diplômes.

Nous retenons pour cela six niveaux de diplômes de l'enseignement supérieur :

- premier cycle (DEUG, DUT, DEUST) ;

- second cycle ;

- troisième cycle ;

- grandes écoles (Ecole de commerce ; Ecole d'ingénieur);

- baccalauréat santé - social ;

- premier cycle non validé.

Pour mesurer l'influence de chaque niveau de diplôme, nous généralisons la régression classique fondée sur le calcul des moindres carrés à des variables qualitatives, ici le diplôme possédé. Pour cela, des variables indicatrices prenant la valeur 0 ou 1 sont créées et ce, pour les différentes modalités de la variable diplôme. Si l'individu considéré possède un diplôme de 3ème cycle, la variable sera codée 1 pour la modalité « $3^{\text {ème }}$ cycle » et 0 pour les autres niveaux de diplôme. Les résultats de l'estimation sont donnés en fixant une des modalités de la variable en référence.

L'estimation (tableau 2) montre une influence positive des niveaux de diplôme de $3^{\text {ème }}$ cycle et des grandes écoles sur le niveau de salaire détenu et une influence négative des autres diplômes ce qui tend à confirmer l'analyse de Spence.

Toutefois, bien que le modèle soit correct $\left(\mathrm{R}^{2}=0,32\right)$, il faut souligner le fait que $70 \%$ du salaire d'un individu est expliqué par d'autres facteurs que le diplôme qu'il possède. 
Tableau 2. Evaluation du modèle de Spence

\begin{tabular}{|c|c|c|c|c|c|}
\hline \multicolumn{6}{|l|}{ ESTIMATION / COEFFICIENTS } \\
\hline \multicolumn{6}{|c|}{ AJUSTEMENT DES MOINDRES CARRES (AVEC TERME CONSTANT) } \\
\hline \multicolumn{6}{|c|}{12730 INDIVIDUS, 6 PARAMETRES (CONSTANTE EN QUEUE) . } \\
\hline IDEN LIBELLE & COEFFICIENT & $\begin{array}{c}\text { ECART } \\
\text {-TYPE }\end{array}$ & STUDENT & PROBA. & V.TEST \\
\hline \multicolumn{6}{|l|}{ CRITERE ( $)$} \\
\hline AF_1 - 3ème cycle & 303.7080 & 10.406 & 29.187 & 0.000 & 28.71 \\
\hline $\mathrm{AF}_{-} 2$ - Bac Santé social & -91.9549 & 10.333 & 8.899 & 0.000 & -8.88 \\
\hline $\mathrm{AF}^{-} 3-\mathrm{Bac}+1+2$ diplome & -266.6343 & 6.311 & 42.246 & 0.000 & -40.87 \\
\hline $\mathrm{AF}_{-}^{-} 4-\mathrm{Bac}+1+2$ non dip & -389.2516 & 7.361 & 52.882 & 0.000 & $-50 \cdot 28$ \\
\hline AF_5 - Grande Ecole & 564.9465 & 11.402 & 49.546 & 0.000 & 47.38 \\
\hline $\mathrm{AF}^{-} 6$ - Second cycle & -120.8137 & 7.786 & 15.516 & 0.000 & -15.44 \\
\hline COÑSTANTE & 1478.4875 & 4.081 & 362.259 & 0.000 & 175.69 \\
\hline \multicolumn{6}{|l|}{ TEST D'AJUSTEMENT GLOBAL } \\
\hline \multicolumn{6}{|c|}{ SOMME DES CARRES DES ECARTS $\ldots \ldots \ldots \ldots . S C E=1969392256.0000$} \\
\hline \multicolumn{6}{|c|}{ COEFFICIENT DE CORRELATION MULTIPLE ... R = $0.5642 \quad \mathrm{R}^{2}=$} \\
\hline \multicolumn{6}{|c|}{ VARIANCE ESTIMEE DES RESIDUS $\ldots \ldots . \quad S^{2}=54777.7656 \quad S$} \\
\hline \multicolumn{6}{|c|}{ TEST DE NULLITE SIMULTANEE DES COEFFICIENTS DES 5 VARIABLES : } \\
\hline FISHER $=1188.569$ & $\mathrm{DEG} \cdot \mathrm{LIB}=5127$ & 724 & & & \\
\hline P.CRIT $=0.0000$ & $\mathrm{~V} \cdot \mathrm{TEST}=69.6$ & & & & \\
\hline
\end{tabular}

\subsection{Evaluation empirique de la théorie du parchemin de Berg.}

Pour Berg, à niveau d'étude identique, il existe une différence de salaire entre les individus qui ont échoué à l'examen et ceux qui ont obtenu le diplôme correspondant. Pour tester cette hypothèse, nous considérons les individus ayant suivi un premier cycle après l'obtention du baccalauréat. Cet ensemble d'individus se partage en deux sous populations :

- Population $1:$ les individus diplômés de premier cycle ;

- Population 2 : les individus non diplômés ;

Afin de vérifier s'il existe une différence significative dans les rémunérations de ces deux sous populations, nous procédons à un test de Darmois (1934) de comparaison des moyennes de deux échantillons quand les variances sont inconnues, soit encore :

$$
\left\{\begin{array}{l}
H o: m_{1}=m_{2} \Leftrightarrow m_{1}-m_{2}=0 \\
H_{1}: m_{1}-m_{2} \neq 0
\end{array}\right.
$$

Avec $m_{1}$ et $m_{2}$ les moyennes respectives des échantillons 1 et 2 .

Le test nous conduit à rejeter l'hypothèse nulle Ho selon laquelle il n'existe pas de différence significative de salaire entre individus diplômés et non diplômés. L'hypothèse de Berg semble donc vérifiée. La possession du diplôme est un des déterminants du salaire d'un individu. 


\section{CONCLUSION}

A la lecture de cet article, le capital humain peut être défini comme les capacités, la connaissance et les compétences incorporées dans les personnes et acquises par l'éducation, la formation et l'expérience. De nombreux théoriciens ont ainsi émis l'hypothèse selon laquelle les individus font un investissement en capital humain dès qu'ils effectuent des dépenses destinées à améliorer leur productivité future. Toutefois, cet enchaînement causal éducation - productivité - gain prôné par la théorie du capital humain a suscité de nombreuses controverses et donné naissance à des théories complémentaires.

Notre comparaison des divers modèles nous a permis de montrer que, pour le cas de la génération 98 en France, les années de scolarité (Mincer, 1958, 1974) jouaient un rôle plus important dans l'explication du salaire reçu, que la place de l'individu au sein de sa génération (Arrow, 1973). Toutefois, il apparaît qu'à niveau d'étude identique, il existe des différences de salaires entre les individus qui possèdent le diplôme et ceux qui ne l'ont pas (vérification de la théorie de Berg, 1970). Nos recherches futures auront pour ambition de valider ou d'infirmer ces premiers résultats !

\section{BIBLIOGRAPHIE}

ABRAHAMS, K. \& MEDOFF, J. (1980). "Experience, Performances and Earnings", Quarterly Journal of Economics, 95, December, pp. 703-736.

ABRAHAMS, K. \& MEDOFF, J. (1981). "Are those Paid more Really more Productive? The Case of Experience", Journal of Human Resources, 16, pp. 182- 216.

AKERLOF, G. (1982). "Labour Contracts as Partial Gifts Exchange", Quarterly Journal of Economics, 92, pp. 543-569.

AKERLOF, G. (1984). "Gift Exchange and Efficiency Wage Theory : Four Views", American Economic Review, 74, pp. 79-83.

ANDERSON, C. A. (1983). "Social Selection in Education and Economic Development", Banque Mondiale, Washington DC.

ARROW, K. J. (1962). "The Economic Implications of Learning by Doing", Review of Economic Studies, 80, pp. 153-173.

ARROW, K. J. (1973). "Higher Education as the Filter", Journal of Public Economics, 2, pp. 193-216.

BECKER, G. S. (1962). "Investment in Human Capital : a Theorical Analysis", Journal of Political Economy, 70, pp. 9-49.

BECKER, G. S. (1964). Human Capital. A Theoretical and Empirical Analysis with Special Reference to Education, Columbia University Press, New York.

BECKER G.S. (1975). Human Capital, 2ème éd. Chicago, University of Chicago Press.

BECKER G.S. (1985). "Human Capital, Effort and Sexual Division of Labour", Journal of Labour Economics, pp. 33-58. 
BERG, I. (1970). Education and Jobs : The Great Training Robberry, Har Monsworth, Penguin.

BOWLES, S. (1985). "The Production Process in a Competitive Economy, Walrassian, Neo-hobbesian and Marxian Models", American Economic Review, 75, pp. 16-36.

BOWLES, S. \& GINTIS, H. (1975). "The Problem with Human Capital Theory Marxist Critique", American Economic Review, 65, pp. 74-82.

BOWLES, S. \& GINTIS, H. (1976). Education and Capitalism in the U.S. NewYork, Basic Books.

CAIN, G. (1976). "The Challenge of Segmented Labour Market Theories to Orthodox Theory: a survey", Journal of Economic Literature, 14, pp. 1215-1257.

CEREQ (2002). Génération 98, Première interrogation, mars 2001.

CONRAD, A. \& MEYER, J. (1957). "Economic Theory, Statistical Inference and Economic History", Journal of Economic History, 17, pp. 524-544.

DALY, A. (1982). "The contribution of Education to Economic Growth in Britain: A Note on the Evidence", National Institute Economic Review, 101, pp. 48-56.

DARMOIS, G. (1934). Statistique et applications. Paris, A. Colin.

DENISON, E. F. (1962). "Education, economics growth and gaps in information", Journal of Political Economy, 70, pp. 124-128.

DIEBOLT, C. (2014). "La cliométrie de l'éducation en perspective historique et comparative", Studia Oeconomica Posnaniensia, 2, pp. 146-161.

DORINGER, P. B. \& PIORE, M. J. (1971). Internal Labour Markets and Manpower Analysis, New-York, Sharpe.

EICHER, J.C. \& MINGAT, A. (1982), "Higher Education and Employment Markets in France", Higher Education, 11, pp. 211-220.

FILER, R. K. (1986). "The Role of Personality and Tastes in Determining Occupational Structure”, Industrial and Labour Relations Review, 39, pp. 412-424.

GOLDIN, C. (1986). "Monitoring Costs and Occupational Segregation by Sex: a Historical analysis", Journal of Labour Economics, 4, pp. 1-27.

GRILICHES, Z. (1970). "Notes on the Role of Education in Production Functions and Growth Accounting", in W. Hanoln, Education, Income and Human Capital in Income and Wealth, 35, Columbia University Press, New York.

GURGAND, M. (1993) "Education et production agricole en Côte d'Ivoire", Revue d'Economie et du Développement, 4, pp. 37-53.

HARTOG, J. (1987). "Earnings Functions: Beyond Human Capital”, Applied Economics, 28, pp. 1291-1309.

JAMISON, D. T. \& LAU, J. (1982). Farmer Education and Farm Efficiency, Baltimore, J. Hopkins University Press.

JAROUSSE, J-P \& MINGAT A. (1986). "Un réexamen du modèle de Mincer", Revue Economique, 6, pp. 999-1031.

KRUGER, A. (1968). "Factor Endowments and Per Capita Income Differences Among Countries", Economic Journal, 78, pp. 641-659.

LAYARD, R., SARGAN, J., AGER, M. \& JONES, D. (1971). Qualified Manpower and Economic Performance. Allen Lane, The Penguin Press, London. 
LAZEAR, E. P. \& MOORE, R. L. (1984). "Incentives, Productivity and Labour Contracts", Quarterly Journal of Economics, 77, pp. 275-295.

LEIBENSTEIN H. (1957). Economic Backwardness and Economic Growth, Wiley (éd.), New York.

LOCKHEED, M., JAMISON, D, LAU, L. (1980). "Farmer Education and Farm Efficiency", Economic Development and Cultural Change, 29, pp. 36-76.

MAGLEN, L.R. (1990). "Challenging the Human Capital Orthodoxy: The Education Productivity Link Re-examined”, Economic Record, 195, pp. 281-294.

MARANTO, C. \& RODGERS, R. (1984). "Does Work Experience Increase Productivity ? A Test of on The Job Training Hypothesis", Journal of Human Resources, 19, pp. 341-357.

MAXWELL, N. L. (1987). "Occupational Differences in the Determination of U.S. Workers Earnings: Both the Human Capital and Structured Labour Market Hypothesis are Useful in analysis", American Journal of Economics and Sociology, 46, pp. 431-445.

MINCER, J. (1958). “ Investment in human capital and personal income distribution ", The Journal of Political Economy, 66, pp. 281-302.

MINCER, J. (1974). Schooling, Experience and Earnings, Columbia University Press, New York.

MIRRLEES, J. (1975). "A pure theory of underdeveloped countries", in Reynolds (eds.), Agriculture in development theory, New Haven, Yale University Press, pp. 84-106.

MOOK, P.R. (1981). "Education and Technical Efficiency in Small-Farm Production", Economic Development and Cultural Change, 19, pp. 723-739.

PERROT, A. \& ZYLBERBERG, A. (1989). " Salaire d'efficience et dualisme du marché du travail ", Revue Economique, pp. 5-20.

PHELPS, E. (1972). "The statistical theory of racism and sexism", American Economic Review, 62, pp. 659-661.

PLANT, M. \& WELCH, F. (1984). "Measuring the Impact of Education on Productivity", in Dean, E., Education and Economic Productivity, Ballinger, Cambridge, Massachusetts.

PLASSARD, J. M. \& TAHAR, G. (1990). "La théorie du salaire d'efficience et des disparités non compensatrices : évaluation à partir de l'enquête FQP", Economie et Prévision, 92-93, pp. 67-75.

PSACHAROPOULOS, G. (1973). Returns to Education : An International Comparison, Amsterdam, Elsevier.

PSACHAROPOULOS, G. (1984). "The Contribution of Education to Economic Growth : International Comparison", in J. Kendrick, International Productivity Comparisons and the Causes of Slowdown, Ballinger, Cambridge, Massachusetts.

PSACHAROPOULOS, G. et WOODHALL, M. (1988). L'éducation pour le développement. Une analyse des choix d'investissement, Paris, Economica.

RAO, M.J. \& DATTA, R.C. (1985). "Human Capital and Hierarchy", Economics of Education Review, 4, pp. 67-76. 
RUMBERGER, R. (1987). "The impact of surplus schooling on productivity and earnings", Journal of Human Resources, 22, p. 24-50.

SCHULTZ, T. W. (1961). "Investment in Human Capital", American Economic Review, 51, pp. 1-17.

SCHULTZ, T. W. (1962). "Reflections on Investment in Man", Journal of Political Economy, 70, pp. 1-8.

SELOWSKY, M. (1969). "On the Measurement of Education's Contribution to Economic Growth", Quarterly Journal of Economics, 83, pp. 449-463.

SOFER, C. (1990). "La répartition des emplois par sexe : capital humain ou discrimination", Economie et Prévision, 92-93, pp. 77-85.

SPENCE, M. (1973). "Job Market Signalling”, Quarterly Journal of Economics, 87, pp. 355-374.

THUROW, C. L. (1972). "Education and Economic Equality", The Public Interest, 28, pp. 66-81.

THUROW, C. L. (1975). Generating Inequality : Mechanisms of Distribution in the US Economy. Basic Books, New York.

WAGNER, J. (1990). "Le test des fonctions de gains : résultats pour cinq pays", Economie et Prévision, 92-93, pp. 61-64.

WEISS, A. (1988). "High School Graduation. Performance and Wages", Journal of Political Economy, 96, pp. 785-820. 